\title{
Experimental Analysis of Cable Distance Effect on Signal Attenuation in Single and Multimode Fiber Optics
}

\author{
Uzairue Stanley ${ }^{1}$, Victor Matthews Olu $^{2}$, Charles Ochonogor ${ }^{3}$, Amaize Peter ${ }^{4}$, Anyasi Francis ${ }^{5}$ \\ ${ }_{1,2,4}$ Electrical and Information Engineering, Covenant University, Nigeria \\ ${ }^{3}$ Electrical and Electronics Engineering, Petroleum Training Institute Warri, Delta State, Nigeria \\ ${ }^{5}$ Electrical and Electronics Engineering, Ambrose Alli University, Ekpoma Edo State, Nigeria
}

\begin{tabular}{l} 
Article Info \\
\hline Article history: \\
Received Feb 17, 2018 \\
Revised May 2, 2018 \\
Accepted May 10, 2018 \\
\hline
\end{tabular}

Keyword:

BTS

Fiber optic

Multimode fiber

Optical amplifier

Single mode fiber

\begin{abstract}
Losses during transmission and high demand of high data rate by the end users have become the biggest challenges facing the telecommunication industries worldwide with Nigeria inclusive. Fiber optic cable as a channel of communication has been adapted worldwide in solving these problems but there is a little limitation in the place of multimode fiber in long distance communication. This paper focuses on the effect of changes in distance on transmitted bandwidth on single mode and multimode fiber. Two cases were considered during this research; (a) with optical amplifier placed in between multimode fiber and (b) without optical amplifier in between multimode fiber. Readings were taken at various distances when specific bandwidth ranging from $50 \mathrm{Mbps}$ to $500 \mathrm{Mbps}$ was transmitted from the base station to the various distances and it was observed that there was no significant changes in bandwidth received at specified distances (100, 200, 300, 400, 500 etc) $\mathrm{m}$ when using single mode fiber, there was a drastic reduction in bandwidth when it get to a distance of $300 \mathrm{~m}$ when using multimode. When optical amplifier was placed in between the multimode fiber at some selected distances after $400 \mathrm{~m}$ from the transmitting BTS, it was noticed that the drastic reduction in transmitted bandwidth was almost eliminated, thereby proven that multimode fiber can be use in long distance communication provided optical amplifiers are incorporated in between the distance to bust the signal strength.
\end{abstract}

Copyright $@ 2018$ Institute of Advanced Engineering and Science. All rights reserved.

\section{Corresponding Author:}

Uzairue Stanley Idiake,

Department of Electrical and Information Engineering,

Covenant University,

KM 10, Idiroko Road, Ota Ogun State, Nigeria.

Email: Stanley.uzairue@ covenantuniversity.edu.ng

\section{INTRODUCTION}

The world is fast becoming a global village and as such the most important tools to make the world become a global smart city are Telecommunications of which internet is the major factor to achieve this. Growth in population also demands growth in the demand of high speed internet. The primary driver to achieve this new access technology which gives true experience of true broadband is high speed internet service and as we all know the most reliable way to get a very high and limitless internet service is through fiber network. Fiber is the proof solution for providing broadband service such as video on demand, online gaming, HD TV and voice over the internet protocol (VOIP) [1]. Since 1970s when fiber optics was invented, the demand and use of fiber optics has grown tremendously. The use of fiber optics has become uncountable and the users have found it more reliable than any other means of data transmission [2]. With the high demand of information traffic due to the Internet, electronic commerce, computer networks, multimedia, voice, data, and video, there comes a high need for a transmission medium with the bandwidth capabilities 
for carrying such high amounts of information is paramount. Fiber optics, with its comparatively infinite bandwidth, has proven to be the solution [3]. Fiber optics has been widely adopted by almost all the telecommunication industries in the world with Nigeria inclusive, some of the front runners in this aspect are as follows; AT \& T, MCI and U.S. Sprint while in Nigeria we have the like of SWIFT Networks, MTN Nigeria, Global, Mainone cable etc, these companies have used [4] and are still using fiber optics to provide the following value added services such as voice over internet protocol, local telephone services with internet services inclusive from their central office switches to various locations and enterprise offices. Fiber optics have become the most adopted means of transmitting data from the internet service provider's office or BTS to any organization such as banks, universities, malls, wall street firms and to other private owned firms who need their services [5]. These firms need secured, reliable systems to transfer computer and monetary information between buildings, to the desktop terminal or computer, and around the world. The security inherent in optical fiber systems is a major benefit [6]. Cable television or community antenna television (CATV) companies also find fiber useful for video services. The high information-carrying capacity or bandwidth of fiber makes it the perfect choice for transmitting signals to subscribers. But in this paper, we shall discuss and focus more on multimode fiber which is the second type of fiber and it is often used in short distance communication such as metro fiber deployment [7].

\section{SOME HISTORICAL BACKGROUND ON FIBER OPTICS IN COMMUNICATION [6]}

In 1966 Kao \& Hockham suggested the use of optical fiber, Corning Glass optical fiber with $20 \mathrm{~dB} / \mathrm{km}$ near $1 \mu \mathrm{m}$ was invented in 1970 following by semiconductor laser with $\mathrm{CW}$ operation at room temperature also in 1970. It was later in 1980 onwards wide spread use of optical fiber communication using SMF and MMF started while in 1990, optical amplification (for increase repeater spacing) was first used and later in 2001 wavelength-division multiplexing (WDM) for increase data rate was used [8]. Fiber optics cable are used for communication system in place of electrical cables because of the following; Electrical communication systems are affected by EMI, Electrical communication systems are associated with low bandwidth ( $4 \mathrm{kHZ}$ - telephone, $100-500 \mathrm{MHz}$ per $\mathrm{km}$ - Coaxial cable), Electrical communication systems are known for high attenuation (20Db/km - typically), [9] Electrical communication systems are known for high cost, this high cost is due to high cost of too many repeaters for a given bandwidth/data rate. For example, 32 channels $(2.048 \mathrm{Mbps})$ PCM link will require one repeater every $2 \mathrm{~km}$, Electrical communication systems are prone to tapping, and Electrical communication systems are bulky in nature [10], [11]. Reference [12]-[14], stated that fiber optics communication have brought about high speed communication which has directly affected the growth of industries directly.

Figure 1, show the diagrammatical representation of optical fiber communication system which consist of the following component or system

Transmitter: fiber optic communicate signal via LED (light communication)

Optical fiber: Fiber optic is divided into two namely; single mode fiber and multimode fiber. These will be explained later in this paper.

Receiver: it uses the process of photo detector to receive signal.

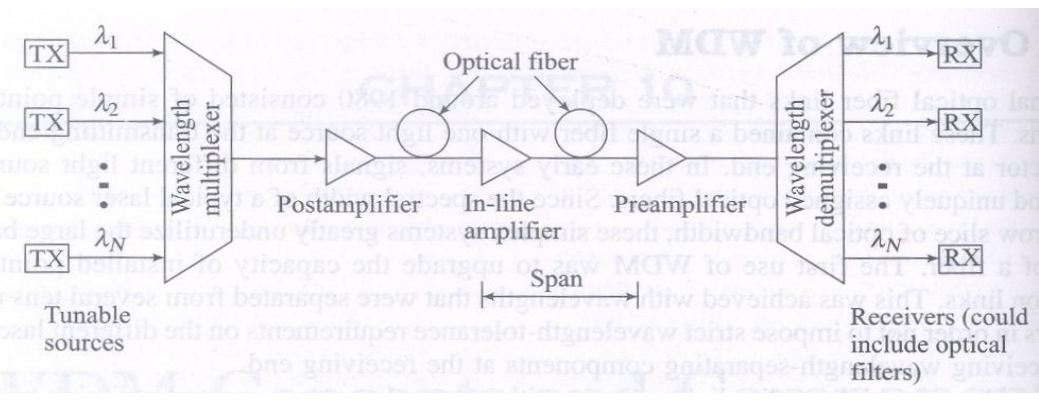

Figure 1. Diagrammatical Representation of Optical Fiber Communication System [1]

\section{RESEARCH METHOD}

3.1. Stages for the design of backhaul link (point to point) using fiber optic

Before designing a long-distance fiber optic network, the following must be put into consideration. 
a. Survey must be done in order to know what is needed for carrying out the project, some of the parameters to be noted during survey include; the distance between the client end and the base station (BTS), where the network room or data centre will be located, the number of persons intended to use the network when completed and if there will be any other value-added services required by the client.

b. Submission of quotation and network diagram by the fiber team, this is to enable the project team analyze the design and quotations to know the most suitable one for the project.

c. Awarding of project

d. Monitoring of the project from starting to finish

e. Commissioning

\subsection{Design methodologies}

Backhaul fiber design between two locations.

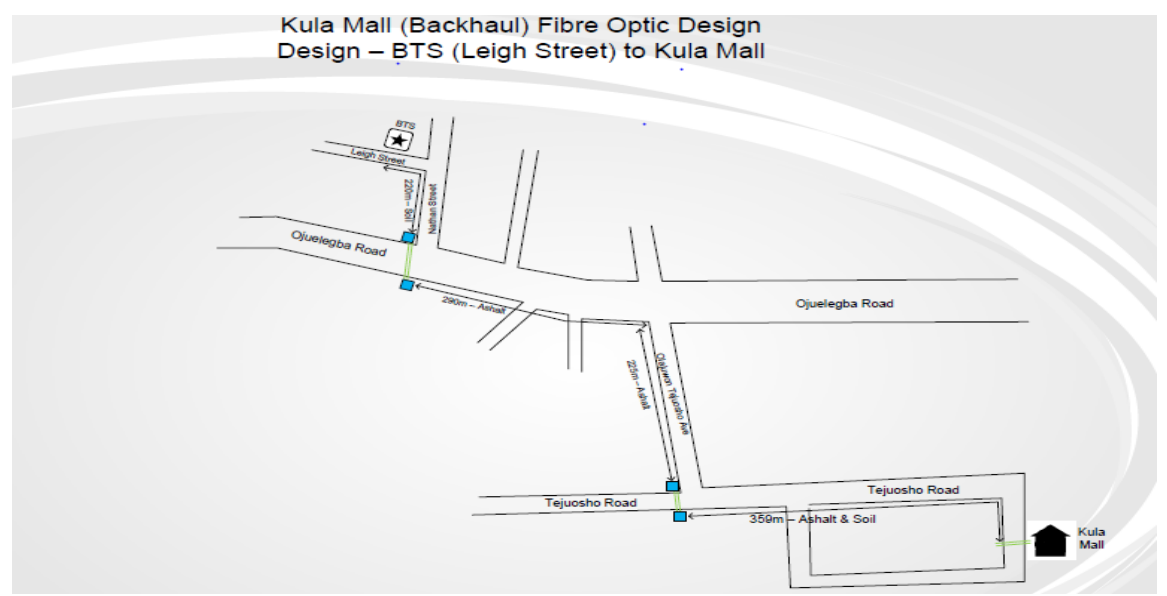

Figure 2. Fiber backhaul connection

\section{DESIGN ANALYSES}

The diagram above (Figure 2) shows the schematic diagram of a fiber backhaul link between two locations which are the BTS and the client end as indicated in the diagram. There are four hand holes between the base station and the client location which is about $5000 \mathrm{~m}$ from the BTS. Single mode fiber and multimode fiber were used in this project to check the variance in the signal strength with increase in distance, the purpose of the four hand holes between the client location and the BTS is to drop some fiber cores at each hand hole so as to create room for future clients within the area, this save cost for the telecommunication company and the clients since the fiber can be taken from the nearest hand hole to the client instead of going a long distance from the BTS.

Single mode fiber and multimode fiber of length $5000 \mathrm{~m}$ was used from the base station to the client end, the signal strength was collected at interval of 100, 200, 300, 400, 500, 600, 700, 800, 900, 1000 all in meters.

At 300 meters when the signal was noticed to start reducing, an optical amplifier was introduced to strengthen the signal and at 700 meters another optical fiber amplifier was also introduced. Bandwidth of 50, $100,150,200,250,300,350,400$ and 500 all in mega bit per second was transmitted from the BTS and signal strength was recorded at each distance, see Table 1 and Table 2 as shown in data presentation.

\subsection{Network room}

The diagram in Figure 3 shows the network room diagram of a particular client, the diagram shows the schematic representation of the internal network diagram of the client. The symbols represented are explained as follows;

a. 6: This represent the router at the base station where the link configurations are carried out by the internet service provider, the bandwidth, the VLANS and the IP address are all configured and assign to the client for internet access. 
b. 1: This is the router at the network room where the internet coming from the BTS is terminated, this router also carries same configuration with the one at the BTS.

c. 2: This is the cisco switch where the internet from router 2 is been re-distributed into the number of offices as requested by the client. And each port on the switch is configured on different VLANs and these VLANs are linked to the IP addresses as assigned to each office in the router configuration.

d. 3: This is the Ethernet patch panel; this is to make a proper arrangement of the UTP cabling during the installation of the network and it also make troubleshooting easy whenever there is fault.

e. 4: This is the media converter chassis where the fiber optic coming from the BTS is been terminated before transferring it to router 1 at the network room. This conversion is necessary because network is being transmitted in the form of light which need to be converted to internet.

f. 5: Fiber patch panel; Fiber optic cable from each office are been spliced at this point and linked with patch cord to the media converter chassis in order to supply internet to the client's office.

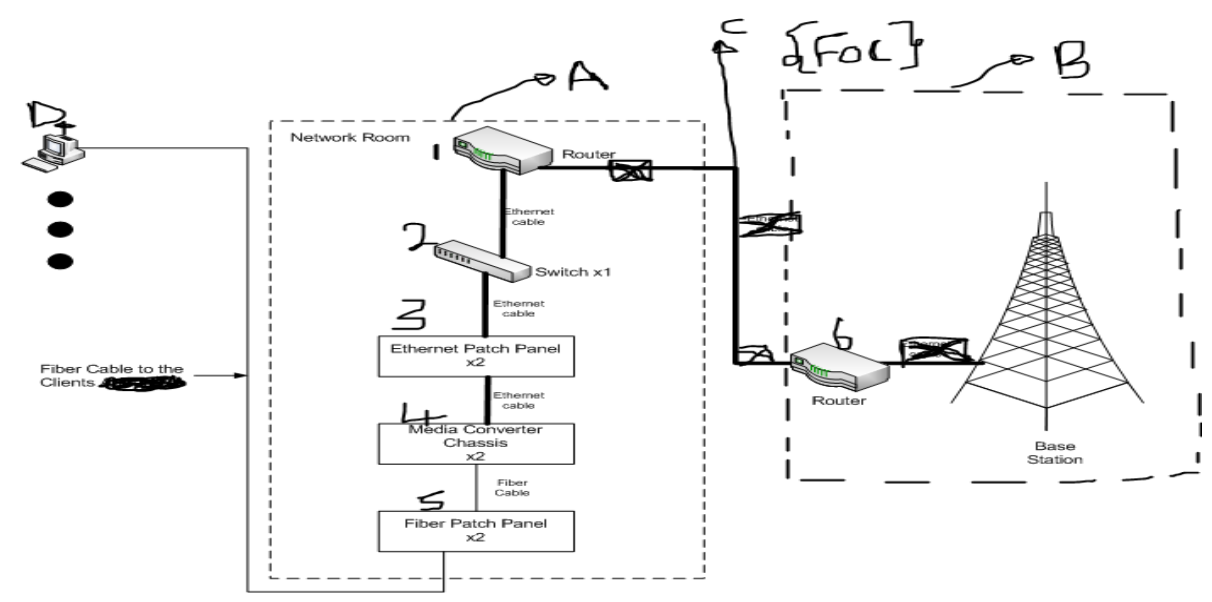

Figure 3. Diagrammatic representation of a typical network room

\subsection{Data presentation}

Table 1. Data without Optical Amplifier in Multimode Fiber

\begin{tabular}{ccccc}
\hline S/N & DIS(m) & Bandwidth TX (Mbps) at the BTS & $\begin{array}{c}\text { Bandwidth RX for Single } \\
\text { Mode Fiber (Mbps) }\end{array}$ & $\begin{array}{c}\text { Bandwidth RX for Multimode } \\
\text { Fiber (Mbps) }\end{array}$ \\
\hline 1 & 100 & 50 & 49.3 & 49.2 \\
2 & 200 & 100 & 99.8 & 99.6 \\
3 & 300 & 150 & 149.9 & 149.5 \\
4 & 400 & 200 & 199.7 & 22.3 \\
5 & 500 & 250 & 249.6 & 260.0 \\
6 & 600 & 300 & 299.5 & 300.8 \\
7 & 700 & 350 & 349.9 & 310.6 \\
8 & 800 & 400 & 399.1 & 399.7 \\
9 & 900 & 450 & 449.8 & 380.9 \\
10 & 1000 & 500 & 499.9 & \\
\hline
\end{tabular}

Table 2. Data with Optical Amplifier in Multimode Fiber

\begin{tabular}{ccccc}
\hline S/N & DIS $(\mathrm{m})$ & Bandwidth TX (Mbps) at the BTS & $\begin{array}{c}\text { Bandwidth RX for Single } \\
\text { Mode Fiber (Mbps) }\end{array}$ & $\begin{array}{c}\text { Bandwidth RX for Multimode } \\
\text { Fiber (Mbps) }\end{array}$ \\
\hline 1 & 100 & 50 & 49.3 & 49.6 \\
2 & 200 & 100 & 99.8 & 99.8 \\
3 & 300 & 150 & 149.9 & 149.8 \\
4 & 400 & 200 & 199.7 & 197 \\
5 & 500 & 250 & 249.6 & 249.9 \\
6 & 600 & 300 & 299.5 & 299.6 \\
7 & 700 & 350 & 349.9 & 349.9 \\
8 & 800 & 400 & 399.1 & 398.9 \\
9 & 900 & 450 & 449.8 & 449.5 \\
10 & 1000 & 500 & 499.9 & 499.8 \\
\hline
\end{tabular}




\section{GRAPHICAL REPRESENTATIONS OF THE DATA COLLECTED}

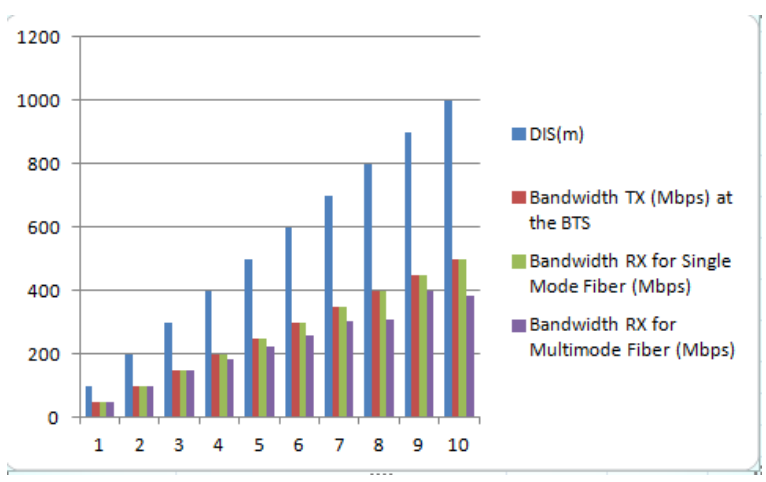

Figure 4. Without Optical Amplifier

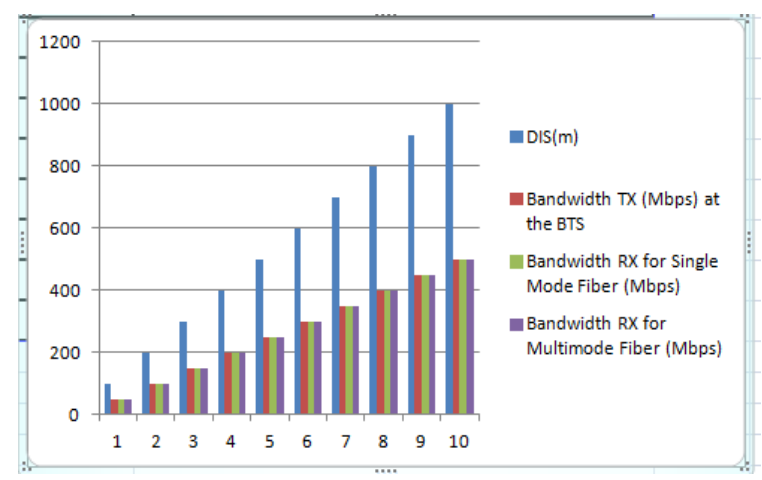

Figure 5. With Optical Amplifier

\section{RESULT DISCUSSIONS}

The result can be discussed in two cases, with optical amplifier in-between multimode fiber and without optical amplifier in-between multimode fiber.

Case one without optical amplifier: from Table 1 and Figure 4 , it was observed that the bandwidth transmitted during this experiment decreases dramatically from distance 400 meters to 1000 meters from the transmitting base station while there was no significant decrease between 100 meters to 300 meters from transmitting base station when using multimode fiber optic cable. But reverse was the case when using single mode fiber optic cable, it was noticed that there was no significant decrease in bandwidth received at the various distances unlike when using multimode fiber.

Case two with optical amplifier: from Table 2 and Figure 5, it was observed that there were no significant changes in signal strength when using either single mode or multimode fiber at all distances. This was due to the present of optical amplifier installed in-between multimode fiber to boost the signal.

\section{CONCLUSIONS}

This has shown that multimode fiber fade with increase in distance and as such not suitable for long distance communication that is above 300 meters long and if it must be use in long distance communication optical amplifier must be install at every interval in order to prevent fading/attenuation but single mode fiber is suitable for both long and short distance communication.

\section{REFERENCES}

[1] A. Nespola, et al., High-Speed Communications Over Polymer Optical Fibers for In-Building Cabling and Home Networking, IEEE Photonics Journal, vol. 2, no. 3, pp. 347-358, June 2010.

[2] P. Polishuk, "Plastic Optical Fibers Branch Out", IEEE Commn. Mag., Sep.2006, pp. 140-148.

[3] Nobel Lecture, 2009, CK Kao, "Transmission of Light in Fiber for Optical Communication".

[4] Gerd Keiser, Optical Fiber Communications, 4th ed., McGraw-Hill, 2008: Chapter 10.

[5] Daniel Cárdenas, et al., "A Media Converter Prototype for 10-Mb/s Ethernet Transmission Over $425 \mathrm{~m}$ of LargeCore Step-Index Polymer Optical Fiber”, J. Lightw. Technol., vol. 24, no. 12, pp. 2923-4951, Dec. 2006.

[6] Jeff Hecht, "Understanding Fiber Optics", 5th Edition, Prentice Hall, 2006

[7] Joseph C. Palais, "Fiber Optic Communications", 5th Edition, Prentice Hall, 2005

[8] Bogyum KIM, Wonhyung LEE and Jinwoo HAN, "Outside Plant Architecture of Fiber-based Access Network", Digest of the 9th international conference on optical Internet (COIN 2010).

[9] Hadaate Ullah, Ridoanur Rahaman, Shahin Mahmud, "Optical Properties of Cadmium Oxide (CdO) Thin Films", vol. 5, no. 1, January 2017, pp. 81-84.

[10] D. K. Sambariya, Seema Shrangi, "Optimal Design of PID Controller for Load Frequency Control using Harmony Search Algorithm”, vol. 5, no. 1, January 2017, pp. 19-32.

[11] Darif A, et al., "IR-UWB: An Ultra Low Power Consumption Wireless Communication Technologie for WSN", Indonesian Journal of Electrical Engineering. 2014, vol. 12, no. 8, pp. 5699-5708.

[12] Francis Idachaba, et al., "Future Trends in Fiber Optics Communication", Proceedings of the World Congress on Engineering 2014, vol. 1, WCE 2014, July 2-4, 2014. 
[13] Nsikan Nkordeh, et al., "The Nigerian Telecommunication Industry: Analysis of the First Fifteen Years of the Growths and Challenges in the GSM Market (2001-2016)", Proceedings of the World Congress on Engineering and Computer Science 2017, vol. 1, WCECS 2017, October 25-27, 2017

[14] Chung et al., "Bit-error-rate Optimization for CDMA Ultra-wideband System Using Generalized Gaussian Approach", International Journal of Electrical and Computer Engineering (IJECE), vol. 7, no. 5, October 2017, pp. 2661-2673

\section{BIOGRAPHY OF AUTHOR}

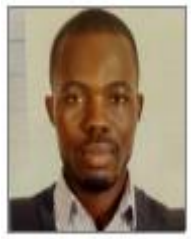

Uzairue Stanley is a $\mathrm{PhD}$ student, lecturer and a researcher in the department of electrical and information engineering, Covenant University, Nigeria. He is a communication expert who has 4years of industrial experience as a telecom engineer. He is a registered engineer by COREN, Member of Nigeria Society of Engineers, Matsers of Engineering in electroctronic and telecommunication engineering, Bachelor degree in electrical and electronics engineering and finally Executve masters in project manage from Cupe Business School, UK. His research interest is in communication engineering, underwater communication, fiber optic network design (fiber optic), Biomedical Engineering etc 